
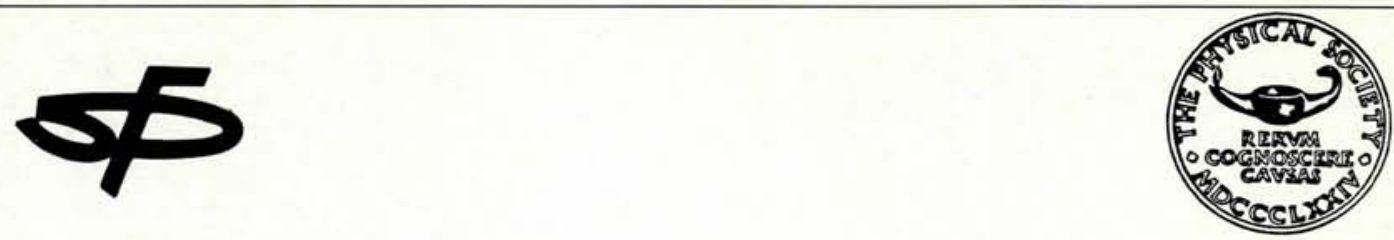

Société Française de Physique

Fondée en 1873

The Physical Society of London

Founded in 1874

\title{
Franco-British Centenary Conference
}

\section{5-9 April 1974, St. Helier, Jersey, Channel Islands}

To mark the 100th Anniversary of the foundations of the French Physical Society (Société Française de Physique) and of The Physical Society of London, a Franco-British Centenary Conference will be held in St. Helier, Jersey, Channel Islands, from 5-9 April 1974.

\section{Plenary Sessions}

In the mornings, lectures will be given, as follows :

Polymeric materials-physics and applications (G. Allen)

Some recent advances in optoelectronics (D.J. Bradley)

Liquid crystals (J.-P. Hurault)

Neutrino physics (A. Lagarrigue)

Some aspects of biophysics (V. Luzzati)

Old and new puzzles about momentum and pseudomomentum (Sir Rudolf Peierls)

The history of The Physical Society (J.A. Ratcliffe)

At one evening lecture, $P$. Aigrain and Sir Brian Flowers will speak on : The financing and administration of physics research : past, present and future. There will also be an evening discussion on physics teaching.

\section{Parallel specialist sessions}

Four subjects have been chosen for parallel specialist sessions atomic physics, nuclear physics, astrophysics, and solid state physics. Further information on these subjects may be obtained from the organising committee conveners below:

Atomic physics (Advances in high resolution spectroscopy. The transfer of spin in collisions)

G.W. Series, J.J. Thomson Physical Laboratory, Whiteknights, Reading, U.K. (telephone: Reading 85123 Extension 385)

Nuclear Physics (Clustering effects in nuclei. High energy methods for probing nuclear structure, with emphasis on mesonic effects. Nuclear sizes and shapes)

Mme H. Faraggi, CEN Saclay, B.P. $N^{\circ} 2$, F-91190, Gif-sur-Yvette, France (telephone : Paris 92088 77)

Astrophysics (Nuclear, atomic and molecular physics in relation to the abundance of the elements in the universe and to other astrophysical problems)
H. van Regemorter, Observatoire de Paris, F-92 Meudon, France (telephone: Paris 6291630 ) for the atomic and molecular physics aspects.

D. Sciama, Department of Astrophysics, South Parks Road, Oxford, U.K. (telephone: Oxford 59878) for nuclear aspects.

Solid State Physics (The metalinsulator transition. Transport phenomena in disordered systems)

B.R. Coles, Department of Physics, Imperial College, London SW7, U.K. (telephone: London 389 5111).

\section{Programme details}

Further details of the programme are now available, including (a) names and subjects of speakers invited to the parallel sessions, (b) full information about the format of the abstracts, (c) registration forms, and (d) room-reservation forms for the Hotel de France, where the Conference will take place.

Please apply directly to The Meetings Officer, The Institute of Physics, 47 Belgrave Square, London SWIX $8 Q X$.

\section{Pramāna}

Commencing in July 1973, Pramana is a new journal of physics published by the Indian Academy of Sciences. In the introductory editorial, $\mathrm{S}$. Ramaseshan justifies the launching on the grounds that a medium was required for the best efforts of Indian physicists. Previously, there had been much unrest because the best papers were published in foreign journals and this delegation of refereeing was leading to loss of judgement and self-confidence, according to Ramaseshan, who is Editor and Chairman of Pramana. Publication of good papers from outside India is "most heartily welcomed". For the time being, Pramana will publish papers in all branches of physics, but it is hoped to branch out into theme journals devoted to Cosmic Physics, Solid State Physics, Molecular Physics, Biophysics, etc.

Each year, there will be two volumes of six issues at $£ 12(\$ 30)$ for libraries and $£ 8(\$ 20)$ for individual physicists.

Enquiries and subscriptions should be sent to S. Arunachalam, Executive Editor, Pramana, Indian Academy of Sciences, Bangalore 560006, India.

Contents of the first two issues of Pramana are set out below :

Atmospheric gamma rays in the energy region 40-1000 GeV (K.C. Anand, R.R. Daniel and S.A. Stephens) Magnetic and electric birefringence in the
isotropic phase of nematic liquid crystals (N.V. Madhusudana and $S$. Chandrasekhar)

High pressure behaviour of liquid caesium (T.G. Ramesh)

Plasmon-magnon interaction in magnetic semiPlasmon-magnon interaction in magnetic
conductors (G. Baskaran and K.P. Sinha)
Off-diagonal long-range order and currents in weakly coupled superconductors (S. Rangarajan and Sudhanshu S. Jha)

Pressure effects on the semiconductor-semimeta transition in $\mathrm{Ti}_{2} \mathrm{O}$, (B. Viswanathan, S. Usha Dev and C.N.R. Rao)

'oca' magnet'c ordering of $\mathrm{Fe}$ impurities in $\mathrm{Pd}_{2} \mathrm{Mn} \mathrm{Sn} \mathrm{(K.R.P.M.} \mathrm{Rao} \mathrm{and} \mathrm{P.K.} \mathrm{lyengar)}$

Ferromagnetic behaviour of interacting superparamagnetic particle aggregates in basaltic rocks (C. Radhakrishnamurty, N.P. Sastry and E.R. Deutsch)

Scaling in prong-number distribution in $\bar{p} p$ collisions (I. Rama Rao and K.V. L. Sarma) Electromagnetic properties of low lying levels in ${ }^{75}$ As (R.C. Chopra and P.N. Tandon)

Photoelasticity in polycrystalline aggregates (G.S. Ranganath and $S$. Ramaseshan)

Stimulated emission of $x$-rays from plasmas generated by short-pulse-laser-heating of solid targets (Sudhanshu S. Jha)

A three dimensional ferromagnetic Ising afluid model (N. Kumar and K.B. Athreya)

Determination of bond lengths in some compounds of rhenium from the $\mathrm{x}$-ray $\mathrm{L} \mathrm{m}$ absorption discontinuity (A.V. Pendharkar and Chintaman Mande)

The law of rectilinear diameter for the liquid-gas phase transition (A.C. Biswas)

Characteristics of a neutron moisture gauge with a solid state detector (M.K. Nagpal, P.P. Mehta, K.K. Nagpal and Rama 